Darton, R.C., Zhang, W. and Taylor, P.H.

Oscillation of aerated liquid on perforated plates and flow-induced vibration of column trays.

Chemical Engineering Research and Design, July 2015, Pages 49-57

doi: 10 1016/j.cherd.2015.03.023 


\title{
Oscillation of aerated liquid on perforated plates and flow-induced vibration of column trays
}

\author{
R.C. Darton ${ }^{1}$, W. Zhang ${ }^{2}$ and P.H. Taylor ${ }^{1}$ \\ ${ }^{1}$ Department of Engineering Science, University of Oxford, UK \\ ${ }^{2}$ Centre for Offshore Foundation Systems, University of Western Australia, \\ Australia
}

\begin{abstract}
The occasional structural failure of distillation and absorption trays operating at low vapour rates has been attributed in the literature to fatigue of tray parts caused by flow-induced vibration. We suggest that synchronous oscillation of gas flow through tray perforations can occur when the perforations are evenly spaced, and the velocity through them is not too high. The controlling mechanism is that of a Helmholtz resonator, in which an oscillating mass of liquid near to each perforation cyclically exchanges kinetic energy with potential energy (head) in the bulk liquid across the tray. The rate of exchange is limited by the wave speed in the bulk liquid. A correlation scheme is suggested for the oscillation frequency based on literature data. We show that effective elastic constants can be calculated for perforated tray panels, and draw attention to the stress concentration around holes near the tray edge that exposes trays to fatigue cracking during vibration. Vibration analysis of an idealized tray structure identified the first 30 modes with resonant frequencies between 25 and $70 \mathrm{~Hz}$, the range where excitation by flow-induced pressure pulsations is possible. Recommendations are made to avoid vibrational damage caused by such pulsations.
\end{abstract}

\section{Keywords}

Oscillation, vibration, Helmholtz resonance, modal analysis, perforated plate, fatigue

Email address of corresponding author richard.darton@eng.ox.ac.uk 


\section{Oscillation of aerated liquid on perforated plates and flow-induced vibration of column trays}

\section{Introduction}

In 1979, at the $3^{\text {rd }}$ International Symposium on Distillation, Brierley reported incidents at $\mathrm{ICl}$ in which trays were damaged by flow-induced vibration, in severe cases leading to collapse of tray sections and even a cracked column shell (Brierley et al., 1979). Vibration occurred in a number of columns, mostly large (diameters up to $8 \mathrm{~m}$ ) operating at pressures from $68 \mathrm{mbar}$ to $4.1 \mathrm{bar}$. Similarly, cracking of beams and tray panels was reported by Winter (1993), the parts commonly showing evidence of fatigue failure; workers reported that such a column "sounded like a bee-hive" due to parts chattering. Vibration damage mostly occurred at low vapour flow rates, and was experienced with both sieve and valve trays, sometimes after only a few hours of operation. It seems that this vibration only occurs at specific conditions: Brierley et al. commented that "the source of vibration has been observed to move up or down a column with changing heat input to the reboiler." Summers (2005) gave details of eight cases where trays had been damaged by "harmonic vibration", again with both sieve and valve trays, operating around the weep point. Further cases of vibration-induced damage were investigated by Geipel and Wolgast (2014), in large columns operating at low gas hole velocities (< $11 \mathrm{~ms}^{-1}$ ).

Brown (1958) had reported pressure fluctuations in the range 3.3 to $32 \mathrm{~Hz}$ in the chamber below a perforated plate (see Chan and Prince (1966) who produced further data and a theory). Kupferberg and Jameson (1970) found that in a plate with multiple holes, as opposed to a single orifice, the frequency of pressure fluctuations did not equal the bubble formation frequency, so that theory for single bubble formation could not be applied. Regimes of jetting and bubbling at a single orifice were investigated by Muller and Prince (1972). They identified two regimes, likely to occur in commercial trays, in which regular pressure fluctuation in the chamber below the orifice was induced by gas-liquid interaction on the plate. These were the 'imperfect bubble' regime (where bubbles break through the surface before they are fully formed) and 'pulsating jet' at higher gas velocities; both required relatively shallow pools of liquid, not more than $30-40 \mathrm{~mm}$ in depth. Waddington et al. (1974) measured the pressure fluctuations in the vapour spaces above and below an operating sieve tray. They found that whilst these were often broad 
band, random and of low amplitude, at certain conditions the variation of flow through all the holes became synchronized, causing higher amplitude fluctuation in pressure drop.

In research supported by ICI, Priestman et al. (1979) measured pressure fluctuations in the range $12-40 \mathrm{~Hz}$ in an air-water simulator fitted with sieve trays. These mostly occurred at hole velocities below $12 \mathrm{~ms}^{-1}$ and tray liquid heads less than $20 \mathrm{~mm}$. They concluded that regular pressure pulsations could be induced by synchronous bubbling or jetting behaviour (oscillations) at the tray holes, and that the effect of pulsations, perhaps resonating with the tray structure, could be the cause of tray damage.

In our previous work Zhang et al. (2014) correlated the frequency data of Priestman et al. (1979) showing that the Strouhal number $\mathrm{fd} / U$ was a function primarily of the Bond number $\rho_{L} g d^{2} / \gamma$, the geometric ratio $d /(s-d)$ and the the Froude number $U / \sqrt{ }\left(g H_{L}\right)$ :

$$
\frac{f d}{U}=0.005\left(1+\sqrt{\frac{\rho_{L} g d^{2}}{\gamma}}\right)+0.46\left(\frac{d}{s-d}\right) \sqrt{\frac{g H_{L}}{U^{2}}}
$$

where symbols have the meanings given in the Nomenclature. Although neither was varied in the experiments with air and water, both the liquid density $\rho_{L}$ and the surface tension $\gamma$ were included to ensure dimensional consistency. The correlation coefficient between the Strouhal number calculated from equation (1) and the experimental value was $r^{2}=0.974$.

The Strouhal number relates the time scale of gas flow through the tray perforation $(d / \zeta)$ to the time scale of oscillation (1/f). As would be expected, the correlation suggests that this varies with the Froude number - the ratio of gas velocity to the speed of a disturbance in liquid height on the tray $\sqrt{ }\left(g H_{L}\right)$. There is also an influence of the geometrical factor $d /(s-d)$ and the Bond number. The latter indicates the magnitude of gravitational body force relative to surface tension force, and is often associated with the shape of liquid surfaces in droplets, jets and waves.

A mechanism which causes regular oscillation of fluid on distillation and absorption trays is presented in the present work, and an improved correlation of frequency data is developed.

\section{Oscillating liquid and pressure pulsations}

\subsection{Liquid motion}


Brierley et al. (1979) noted from Waddington's work that random bubbling at the tray perforations only turned into synchronized pulsations under specific conditions, and suggested that another mechanism must play an important role, yielding a natural frequency associated with the fluids in the column. They mentioned three attempts to model this frequency, ("Oscillating plug model", "Mass/spring model", "Acoustic resonance model") but none of these had been satisfactory. In particular, models in which pressure energy is stored in a gas reservoir predict frequencies that are much too high.

Both Muller and Prince (1972) and Priestman and Brown (1988) directly observed liquid oscillation at the tray hole. Liquid moves away from the hole horizontally, then flows back towards, and to some extent into it; as the next cycle starts, liquid is blown out of the hole, and away again. During these movements, the hole gas velocity rises and falls, and pressure in the inter-tray spaces above and below the trays varies as well. Working with rectangular sieve trays of dimension $410 \times 405 \mathrm{~mm}$, Priestman et al. (1979) found the frequency of pressure pulsations to increase linearly with the hole velocity but to vary little with hole diameter. Increasing the liquid head on a tray also increased the frequency in most cases. Photography revealed a circular spread of liquid from the hole at each cycle, with a diameter of spread similar to the hole pitch. The pulsation frequency decreased as pitch increased, and they concluded that the hole-edge to hole-edge distance $(s-d)$ was a crucial factor. This key observation suggests a mechanism by which oscillations are synchronized. It implies that adjacent holes interact on a time scale comparable with that of the pressure pulsations $(25-80 \mathrm{~ms})$, which is similar to the time scale on which a variation in liquid head would be communicated between adjacent holes $\left(\sim(s-d) / \sqrt{ }\left(g H_{L}\right)\right)$ where $H_{L}$ is the liquid height on the tray. This is much longer than the time for a pressure disturbance to travel the same distance in the vapour phase $(<0.1 \mathrm{~ms})$. Local variations in pressure in the inter-tray space are evened out rapidly. Thus we would not expect the volume of the inter-tray space to affect the pulsation frequency, and this was confirmed by some additional experiments: a fivefold variation in the inter-tray volume had little effect on the frequency observed (Priestman et al., 1979). For a tray with regular perforation spacing (square or equilateral triangle pattern), the time for communication through the liquid phase between adjacent holes will be the same all over the tray. This will promote the synchronous variation of vapour/liquid interactions at all holes, since where all the perforations have the same diameter and pitch geometry and flow conditions they will pulsate at the same frequency. Very rapid equalisation of pressure in the vapour spaces above and below the tray will 
quickly adjust pulsation at any hole that is temporarily out of synchronization. The expected motion of liquid on the tray and its effect on gas velocity through the holes is illustrated by the sequence of conditions sketched in Figure 1.

Figure 1 Oscillation of liquid at a tray perforation, and between perforations (not drawn to scale)

Figure 1 shows the motion on the tray at a sequence of four moments during an oscillation cycle. Characteristics of these conditions are as follows:

(a) Greatest obstruction of hole by liquid; hole velocity is at a minimum; liquid height between the perforations is a minimum; weeping is possible; pressure under the tray is at its minimum; pressure above the liquid layer is at its maximum.

(b) Hole velocity is increasing; liquid is pushed radially away from the perforations; liquid height between perforations is increasing.

(c) Least obstruction of hole by liquid; hole velocity is at a maximum; liquid height between the perforations is a maximum; weeping is reduced or zero; pressure under the tray is at its maximum; pressure above the liquid layer is at its minimum.

(d) Hole velocity is decreasing; liquid flows back towards the perforations; liquid height between perforations is decreasing. Tray then returns to condition (a).

In Figure 1 the gas flow through the hole is shown as a jet, but a similar sequence of fluid motions will occur if the gas flow is in the form of bubbles. Conditions (a) and (c) will then represent the situation when the volumetric flow-rate of bubbles is a minimum and a maximum respectively. We note that the data of Priestman et al. (1979) show no effect of the transition from bubbling to jetting on the oscillation frequency, when this transition is identified using the method of Lockett (1986, p 35) for example. Priestman and Brown (1981) confirm that "neither the pulsation amplitude nor frequency measurements showed any correlation with the onset or the occurrence of liquid weeping".

In some ways the liquid motion on the tray is similar to that of a standing wave. This occurs when waves are propagated along a channel closed at both ends so that incident waves are reflected. Each end of the channel must correspond with an anti-node (no lateral motion of liquid), so that the channel length is an integral number of half-wavelengths. The liquid surface rises and falls at a frequency $c / \lambda$ where $c$ is the wave speed and $\lambda$ the wavelength. In the case of liquid on a plate where waves are initiated at a 
large number of perforations, trains of waves travelling in opposite directions will meet each other. At the mid-point between two adjacent perforations there will be an anti-node, where the amplitude of vertical movement of the surface is greatest, and where, by symmetry, there is no lateral motion; this is similar to what happens at the ends of a channel with a standing wave. However the motion is not that of a standing wave, because at the perforation there is bubbling or jetting of vapour through the orifice. The liquid there oscillates radially - see Figure 1 - and the pressure is determined by both liquid and gas flow dynamics.

The mechanism determining liquid motion on the tray is that of a Helmholtz resonator, in which an oscillating mass exchanges kinetic energy with a reservoir which can store it as pressure energy. The oscillating mass in this case consists of fluid near to the perforations which possesses nearly all the kinetic energy (radial velocity declines as distance ${ }^{-1}$, and vertical velocities not near the gas flow are very small). As the liquid moves away from the hole during the cycle (Figure 1, (a) $\rightarrow(\mathrm{c})$ ), this energy is stored as an increase in fluid head. In a Helmholtz resonator the frequency of the oscillation is proportional to $c / L_{H}$, where $c$ is the speed of propagation of a change in head (wave velocity), and $L_{H}$ is a composite length scale dependent on the geometry of both the oscillating mass and the energy reservoir. Although the classical Helmholtz resonator model is generally invoked as a description for oscillations such as those induced by blowing across the mouth of a bottle (Morse and Ingard 1968, p. 489-90), it can also be used to describe freesurface oscillations in harbours excited by wave disturbances incident from the open sea (Mei 1989, p.206-212). Then, the mouth of the bottle is replaced by the gap between breakwaters, and the gas in the bottle by the water surface within the harbour. Because the wave motion is restricted to a 2-D free-surface, the 'harbour resonance' becomes a direct analogue for the fluid layer on the tray.

Because the inter-tray space exhibits a uniform pressure and all perforations pulsate synchronously, the force on the tray also oscillates, with the same frequency. The maximum upward force on the tray occurs at the condition shown in Figure 1(c), and the minimum at condition Figure 1(a). This periodic force will cause vibration of tray parts, and if the frequency corresponds to a resonant frequency of the tray, then amplification of the vibration will occur and the result can be catastrophic mechanical failure.

During this fluid oscillation, the pressure is a maximum under a tray when the pressure is a minimum above the tray, and vice versa. 
Consequently, in a stack of similar trays, the oscillation on adjacent trays will be out of phase by exactly $180^{\circ}$.

\subsection{Correlation scheme}

(a) Wave velocity

Lamb (1932) analysed waves travelling on a liquid of mean depth $H_{L}$, whose amplitude is small compared with both depth and wavelength (Airy waves). The velocity is given by

$$
c^{2}=\left\{\frac{g \lambda}{2 \pi}+\frac{2 \pi \gamma}{\rho_{L} \lambda}\right\} \tanh \left(\frac{2 \pi H_{L}}{\lambda}\right)
$$

We note from equation (2) that for long, gravity-driven waves, the velocity is $\sqrt{ }\left(g H_{L}\right)$. For the experiments of Priestman et al. (1979) the surface tension term in (2) generally increases the wave speed by not more than a few percent, whereas the term $\tanh \left(2 \pi H_{L} / \lambda\right)$ can reduce it by up to about $20 \%$ as $H_{L} / \lambda$ increases.

(b) Wavelength

The preliminary correlation, equation (1), shows the importance of hole pitch as a length scale affecting the frequency of oscillation. However the data also demonstrate that there is an influence of hole velocity, and a characteristic dimension of the oscillation is $L$, found to be

$$
L=3.8\left\{s_{m}-0.5 d\left(1-3.1 W e^{-0.5}\right)\right\}
$$

where calculation of the mean pitch $s_{m}$ is described in section 2.3. Equation (3) introduces the Weber number based on the gas density, which relates two surface forces: that due to the dynamic gas pressure at the perforation, and the surface tension. Equation (3) shows that as Weber number increases, the length scale of the oscillation decreases somewhat, perhaps because the equilibrium position of the liquid is pushed away from the perforation. The data can be correlated with a wave speed from (2) and an effective wavelength $\lambda$ given by

$$
\lambda^{-1}=L^{-1}+7.84210^{-4} \mathrm{Bo}^{0.5} \mathrm{Fr} d^{-1}
$$

The effective wavelength varies from about 1 to $3 x$ the hole pitch for these data. 
(c) Frequency

With the wave velocity calculated from equation (2) and the effective wavelength from (3) and (4), the experimental frequency data of Priestman et al. (1979) are correlated by

$$
S t=\frac{f d}{U}=5.537 \frac{d}{\lambda} \frac{c}{U}
$$

Combining equations (4) and (5) yields (6), a form more readily comparable with equation (1), our original correlation,

$$
S t=0.00434 \frac{c}{\sqrt{g H_{L}}} B o^{0.5}+5.537 \frac{d}{L} \frac{c}{U}
$$

The numerical ranges of dimensionless numbers on which this correlation is based are: St 0.02 to 1.1 ; Bo 3.1 to 34.3 ; We 1.3 to 26.5 ; Fr 10.3 to 65.9.

\subsection{Correlation of experimental data}

The synchronous oscillation of fluid occurs because each hole on the tray is separated from its nearest neighbours by the same spacing. An exception will be those holes at panel edges where the adjacent holes may, for construction reasons, not be placed at the usual regular separation. Also, for holes near the wall, weir or inlet downcomer, adjacent holes will be absent on one side. In three of the trays (C, D, E) investigated by Priestman et al. (1979) the number of holes was reduced $(403,256,108)$ whilst keeping the pitch (equilateral triangle pattern) and hole diameter the same. As a result these trays show the effect of leaving an area unperforated: reducing the perforated fraction from $82 \%$ to $20 \%$ reduced the oscillation frequency by about $10 \%$. This is not a strong effect, but it does need to be accounted for.

In our earlier work, Zhang et al. (2014) compensated for the presence of an unperforated zone by adjusting the calculated frequency according to the fraction of the tray unperforated. However here we adopt a different approach. Holes adjacent to an unperforated zone will, on that side, transfer kinetic energy to a larger reservoir than usual; this will tend to increase the length scale $L$ and decrease the frequency. The synchronous nature of the oscillation is maintained by the communicating vapour spaces beneath the tray and above the tray, so the centre of radial oscillation at the edge-holes is displaced towards the unperforated zone with a consequent effect on all other 
holes. All holes experience a slightly longer effective wavelength. We account for this stretching by calculating a mean value of the hole pitch, $s_{m}$, according to an empirical relation

$$
s_{m}=s(0.5+0.5 \alpha)^{-0.5}
$$

where $\alpha$ is the fraction of the plate that is uniformly perforated. This fraction is calculated from the area of plate $A_{p h}$ that would be associated with a single hole in a similarly perforated plate of infinite extent. For holes on an equilateral triangular pattern with pitch $s, A_{p h}$ is $\left(s^{2} \sqrt{3}\right) / 2$. We then have $\alpha=$ $N A_{p h} / A_{p}$, where there are $N$ holes in a plate of area $A_{p}$, and can calculate $s_{m}$ from equation (7). These calculations are shown in Table 1 and 2. (Note that Zhang et al., 2014, used a different calculation method which resulted in higher values of $\alpha$.)

$\begin{array}{lcccccccc}\text { Tray } & \mathrm{A} & \mathrm{B} & \mathrm{C} & \mathrm{H} & \mathrm{I} & \mathrm{L} & \mathrm{N} & \mathrm{O} \\ \text { Number of holes } & 403 & 391 & 403 & 108 & 104 & 108 & 38 & 38 \\ \text { Hole diameter, mm } & 4.76 & 6.35 & 9.53 & 9.53 & 12.3 & 15.87 & 12.3 & 15.87 \\ \text { Hole pitch, mm } & 19.05 & 19.05 & 19.05 & 38.1 & 38.1 & 38.1 & 66.0 & 66.0 \\ \alpha & 0.763 & 0.740 & 0.763 & 0.818 & 0.787 & 0.818 & 0.863 & 0.863 \\ s_{m}, \mathrm{~mm} & 20.29 & 20.42 & 20.29 & 39.97 & 40.31 & 39.97 & 68.38 & 68.38\end{array}$

Table 1. Tray details for eight perforation geometries. Rectangular tray 410x405 mm, Priestman et al. (1979).

Figure 2 shows the Strouhal numbers calculated from the correlation scheme plotted against the experimental values for the trays of Table 1.

Measurements were made either at constant hole velocity, or constant liquid height, as indicated in the key to Figure 2 next to the tray letter A, B, C etc. The new correlation is an improvement compared with that of Zhang et al. (2014), now having a regression coefficient $r^{2}=0.991$.

Figure 2. Correlation of pulsation frequency. Strouhal number calculated from equation (6). Data of Priestman et al. (1979)

The hole diameter and pitch of trays $C, D$ and $E$ were identical, but as $D$ and E contained fewer holes as shown in Table 2, $\alpha$ was reduced. Consequently the mean pitch, $s_{m}$ increased from $20.29 \mathrm{~mm}$ to $22.11 \mathrm{~mm}$ to $24.55 \mathrm{~mm}$ respectively. 


$\begin{array}{lccc}\text { Tray } & \text { C } & \text { D } & \text { E } \\ \text { Number of holes } & 403 & 256 & 108 \\ \text { Hole diameter, mm } & 9.53 & 9.53 & 9.53 \\ \text { Hole pitch, mm } & 19.05 & 19.05 & 19.05 \\ \alpha & 0.763 & 0.485 & 0.204 \\ s_{m}, \mathrm{~mm} & 20.29 & 22.11 & 24.55\end{array}$

Table 2. Tray details for tray series $C, D$ and $E$ with reducing number of holes. Rectangular tray 410x405 mm, Priestman et al. (1979).

Figures 3 and 4 show frequency data for these three trays as the liquid head on the tray was varied at constant hole velocity. Solid lines represent calculations of frequency from the correlation scheme. It can be seen that the use of the mean pitch $s_{m}$ defined by the empirical equation (7) accommodates the effect of the presence of unperforated areas to a large extent, justifying the form of equation (7).

Figure 3. Pulsation frequency for Trays $C, D$ and $E$, at $U=4 \mathrm{~m} / \mathrm{s}$ : data of Priestman et al. (1979), and calculated from equation (6)

Figure 4. Pulsation frequency for Trays $C, D$ and $E$, at $U=8 \mathrm{~m} / \mathrm{s}$ : data of Priestman et al. (1979), and calculated from equation (6)

\section{Pressure pulsations: amplitude}

Pressure pulsations on adjacent trays cannot be in-phase because the tray pressure drop must fluctuate to be consistent with gas flow through the perforations. The pressure in the inter-tray space can be calculated from the unsteady state continuity equation assuming isentropic compression and expansion, and this suggests that the r.m.s. pressure fluctuation $\boldsymbol{p}$ will vary as $\boldsymbol{P} \boldsymbol{\kappa} \boldsymbol{A}_{\boldsymbol{h}} \boldsymbol{u} / \boldsymbol{V} \boldsymbol{f} . \boldsymbol{P}$ is the mean pressure, $\boldsymbol{\kappa}$ the isentropic expansion factor of the gas, $\boldsymbol{A}_{\boldsymbol{h}}$ the total hole area on the tray, and $\boldsymbol{V}$ the volume of the inter-tray space. $\boldsymbol{u}$ is a measure of the variation in hole velocity, and is a fraction of its mean value $\boldsymbol{U}$. At high values of $\boldsymbol{U} / \sqrt{ }\left(\boldsymbol{g} \boldsymbol{H}_{\boldsymbol{L}}\right)$ we expect that $\boldsymbol{u} / \boldsymbol{U} \rightarrow \mathbf{0}$ as a steady jet forms which entrains droplets rather than causing liquid oscillation at the hole, and at low values of $\boldsymbol{U} / \sqrt{ }\left(\boldsymbol{g} \boldsymbol{H}_{L}\right)$ oscillations will be suppressed by heavy weeping. It is not clear however how $\boldsymbol{u}$ will vary in detail, and though data of Priestman et al. (1979) do suggest that the amplitude of the pressure 
pulsations decreases with increasing frequency, the picture is complex, as shown in Figure 5. In this figure, contours of the r.m.s. amplitude of the pressure fluctuation measured by a single transducer underneath the test tray are shown as a function of both head of clear liquid on the tray, and hole velocity, for trays $B, H$ and $L$.

Figure 5. Pressure pulsation fundamental r.m.s. amplitude plots (data of Priestman et al., 1979)

\section{Vibration analysis}

In order to check whether resonance is likely to occur between fluid oscillations and the tray structure, a vibration analysis must be carried out, for which commercial software is available. We have used the finite element package SOLIDWORKS (Dassault Systèmes S.A., 2014). In the analysis we used effective values for the Young's modulus and Poisson's ratio of the perforated plates, calculated by a strategy outlined in Zhang et al. (2014). We assume that the holes are small compared to the plate dimensions so that bending deformation of the plate surface occurs over distances large compared to the hole. It is reasonable, in the calculation, to replace the perforated plate by an equivalent solid one with reduced bending stiffness, at least for determination of global bending and free vibration modes. Even a small hole area ratio (free area) leads to significant reduction in the effective Young's modulus (reduced stiffness of the plates), but the change in the effective Poisson's ratio is much smaller.

These effective elastic moduli are used in the vibration analysis to estimate the resonant frequencies and mode shapes which might be excited, if these modes are long compared to the hole pitch. As an example we considered an idealised tray structure approximately $2 \mathrm{~m} \times 2 \mathrm{~m}$, with panels of width $406 \mathrm{~mm}$ set side by side, joined and supported by small U-angle sections. All the dimensions were chosen to be representative of what would be found in a typical distillation column. The panels were stainless steel, $2 \mathrm{~mm}$ thick with $15 \%$ free area. A vibration analysis of this geometry was conducted with SOLIDWORKS, allowing for the presence of the fluid layer on the tray, which, by adding extra weight, tends to reduce the vibration frequency. The frequencies and associated mode shapes of the first 30 modes were identified, as shown in Figure 6 . These modes occur in the range $25-70 \mathrm{~Hz}$ associated with tray vibration. Results for two cases are shown, the lower 
when the tray perimeter is simply supported (SSSS - restrained against deformation out of plane but no rotational restraint on each edge, which thus acts as a frictionless hinge) and the higher where both translation and rotation at the edge is suppressed (CCCC - all boundaries rigidly clamped). These calculations illustrate that the edge conditions play a relatively minor role - a convenient finding, as in practice the edge conditions are likely to be intermediate between simply supported and rigidly clamped.

The results show that there are many vibration modes in the range of observed tray pressure pulsation frequencies. But, at least for a symmetrical plate layout, only the odd modes are likely to partake in the oscillation. The even modes have equal plate areas moving vertically upwards and downwards simultaneously (in and out of phase), so the net contribution to the oscillation may be small. In contrast the odd modes would be able to participate in the net fluid layer oscillation, with the spatially averaged plate surface having a net upwards and then downwards motion in time. This motion would be accelerated by pressure drop oscillating in-phase. Thus, during operation at conditions which enable coupling between flow-induced pulsations and the structure, and with relatively low structural damping, it would not be surprising if damaging levels of vibration were to occur.

Figure 6. Structural vibration resonance frequencies of idealized tray structure, first thirty modes (Zhang et al., 2014)

Perforated plates will be more flexible than solid plates of the same material, thus at long bending scales compared to the hole spacing the resonant frequencies will be lower. Locally to each hole there will also be a stress concentration effect as the stress field is amplified compared to the area-averaged value. The stress amplification for small free area is $3 x$ (Timoshenko and Goodier, p90) but this grows significantly as the free area increases. Thus, fatigue cracking, running from the boundary supports towards the closest hole and from hole to hole, is a serious risk when perforated plates vibrate.

\section{Discussion and Recommendations}

\subsection{Negative Resistance theory}

An important question for tray designers and column operators is whether destructive vibration of trays can be predicted in advance, so that tray design or operating conditions can be changed so as to avoid it. This question was 
tackled by Brierley et al. (1979) who based their prediction on the idea that "flow instability may occur under conditions where there is a 'negative' resistance to vapour flow", proposed by Waddington (1973). The concept of negative resistance is shown in Figure 7, for a sieve tray. At constant liquid flow-rate, as vapour rate increases, pressure drop across the plate increases, but hold-up of vapour in the liquid also increases whereby the static liquid head can decrease. The result is that the total pressure drop can, in theory, show a minimum, as in Figure 7. However Brierley et al. also point out that in practice, due to weeping, static head falls as vapour rate decreases at low vapour rate, so that the minimum in Figure 7 may not actually occur.

Nevertheless those authors suggested that Waddington's work and their own was "consistent with instability occurring at a region on the pressure drop-flow characteristic having a small or zero slope". Brierley et al. located the turning point, i.e. the 'critical velocity', by differentiating a correlation predicting the total pressure drop. A similar procedure was followed by Lockett (1986, p116) and by Summers (2005). It may also be applied to valve trays, since the region between the closed and open balance points in the dry tray pressure drop characteristic has a very small positive slope (Lockett, 1986, p.82).

Figure 7. Idealised pressure drop characteristic, from Brierley et al. (1979, Fig. 2)

Descriptions in the published literature of exactly what phenomena occur in the region labelled 'Unstable flow regime' in Figure 7 are lacking. But we speculate that if the mean hole vapour velocity is less than the Critical Velocity, there is another possible operating point with the same total pressure drop (to the right of the Critical Velocity in Figure 7). This second operating point represents holes that take a higher vapour rate and experience, locally, a lower static liquid head. For some holes to operate at each condition is not a stable situation, since the overall vapour rate exceeds that supplied to the tray. The result must be then for flow-rate to all holes to decrease and the dry pressure drop to fall. Subsequently the flow-rate will increase again. Possibly some holes will flip from a condition with less than critical flow, to take a flow greater than critical, then back again, with consequent local changes in static head.

This imagined description of a tray instability could account for oscillation of tray pressure drop, coupled to variation in total vapour flow through the perforations. There are some problems however. The change in static liquid head shown in Figure 7 is due to change in gas hold-up, based on 
steady state correlations. It may be doubted whether the hold-up could indeed vary like this at the frequency of $15-70 \mathrm{~Hz}$ typical of tray pulsations. The lower value of static head experienced by those holes operating above the critical velocity means that liquid must be displaced which will flow back when operation of the hole flips to its original sub-critical velocity. This implies liquid motion at the same frequency - certainly possible, but a mechanism driving such regular waves must be part of the theory, and this is missing. Brierley et al. (1979) recognised the need for another mechanism to explain the frequency of oscillation, and in its absence suggested an empirical correlation of plant data $\left(f\left(s^{-1}\right)=31(P, b a r)^{0.3}\right)$ as an interim solution.

In spite of these problems, success is claimed for Negative Resistance theory in setting a reasonable value for a minimum hole velocity to avoid oscillation, a key design parameter. Taking Lockett's estimate, with some typical values for sieve trays that he supplies (Lockett, 1986, p 116) gives

$$
U_{\text {crit }} \sqrt{\rho_{G}}=0.447 \sqrt{\rho_{L}}
$$

For the air-water system at 1 bar, $U_{\text {crit }}$ is $12.9 \mathrm{~ms}^{-1}$ according to equation (8).

Priestman and Brown $(1981,1988)$ suggested an alternative explanation for the condition arising from equation (8), based on a detailed examination of measured pressure traces. They concluded that pulsations would not occur if the hole dynamic head were greater than about $100 \mathrm{Nm}^{-2}$ for the air-water system at 1 bar; this corresponds, also, to a hole velocity of $12.9 \mathrm{~ms}^{-1}$. Priestman and Brown pointed out that this critical value of dynamic head also agreed with data for three commercial sieve trays reported by Brierley et al. (1979).

\subsection{Helmholtz resonance theory}

Our theory that tray oscillations are caused by Helmholtz resonance does not involve the gas hold-up. The kinetic energy of the oscillation resides in the liquid phase situated adjacent to the perforation, and during the oscillation cycle energy is temporarily stored as liquid head by the liquid mass on the tray. The rate at which liquid head is stored, and released, regulated by the wave velocity (equation 2), is a primary factor determining the oscillation frequency.

Our correlation scheme is based upon physical intuition and is dimensionally consistent. But it must be acknowledged that the data on which the correlation is based are for the air-water system at 1 bar, taking values of 
$\rho_{G}, \rho_{L}$ and $\gamma$ respectively as $1.2 \mathrm{kgm}^{-3}, 1000 \mathrm{kgm}^{-3}$ and $0.072 \mathrm{Nm}^{-1}$. As these system properties were not varied, we cannot be sure that they have been correctly incorporated in the correlations, particularly with respect to the densities selected for use in the Bond and Weber numbers. There is a need for more experimental work to verify our correlational approach over a wider range of conditions, and particularly with different vapour/liquid systems.

Our work has focussed on flow-induced oscillations that can resonate with mechanical structures, generally at frequencies of $20 \mathrm{~Hz}$ or more. These should not be confused with lower frequency motions sometimes termed "sloshing" which occur at $5 \mathrm{~Hz}$ or less (Lockett, 1986, p105). This instability involves relatively large amplitude waves of liquid moving across the tray from side to side, as different groups of holes come into operation intermittently. The low frequency of this instability is very unlikely lead to structural resonance and fatigue failure, though it may disrupt operation in other respects, particularly in small columns, as Lockett points out.

Although our correlation is based on experiments with sieve trays, the same mechanism should control potential oscillations on valve trays, with the added complication that at low vapour rates the hole resistance will change as the valves close. This may not however have much effect on the oscillation frequency, which is mainly dependent on fluid flow on the tray. Photographs of valve tray damage due to flow-induced vibration have been presented by Winter (1993) and Geipel and Wolgast (2014). These show a ring of highly polished or worn surfaces adjacent to the tray perforations suggesting that the valve legs and cap have been abrading the surface with high frequency contacts. Winter states that "such polished surfaces are not seen in columns that operate in a stable non-resonant condition".

\subsection{Recommendations to avoid vibration damage}

(a) When specifying a tray design, it should always be stated whether or not trays will be required to operate at significantly reduced throughput for more than very brief periods. Such operation might include frequent start-ups/shutdowns, for example.

(b) Risk factors for damaging tray vibration include:

- long flow path $(>1 \mathrm{~m})$

- low liquid head on tray $(<25 \mathrm{~mm})$

- low hole velocity $\left(0.5 \rho_{G} U^{2}\right)<100 \mathrm{~N} \mathrm{~m}^{-2}$

- tray stacks where conditions vary little from tray to tray (e.g. absorption of trace components, distillation of close-boiling mixtures). 
These are cases where the frequency will be similar in adjacent trays, strengthening the oscillation.

(c) Mechanical design should include enough stiffening to increase the vibration frequency of the structure beyond what is likely to be encountered in flow-induced oscillations.

(d) The modes of structural vibration of trays can be checked using a standard finite element design package. The first mode is particularly important: its frequency should exceed the frequency of any expected oscillation by a safe margin.

(e) Stress concentrates around holes. Care should be taken with the placing of all holes in the perforated plates. Holes, including holes for bolts and other fittings should not be close together as a stress concentration then arises where fatigue cracking can be initiated.

(f) It may be possible to avoid synchronous oscillation at the tray perforations by not placing them all at the same spacing. For example a rectangular layout of perforations could be chosen, to "de-tune" the tray.

(g) The sound of vibration from inside an operating column (buzzing, chattering) should always be investigated immediately, as damage can occur in a few hours, or sometimes quicker. If the sound disappears as vapour throughput is increased, this is an indicator of flow-induced vibration. With valve trays that are in operation it is not normal for the sound of valve chattering to be audible outside the column, above general plant noise.

(h) During periods of generally reduced throughput - slack demand for product, interruption of feedstock supply, etc. - columns and their trays should be checked for the possibility of flow-induced vibration, as they may be operating at conditions not originally envisaged in the design.

\section{Conclusions}

Fatigue cracking and failure of column trays can happen when they vibrate in resonance with pressure pulsations in the inter-tray space. These pressure pulsations occur over a certain range of operating conditions with both sieve and valve trays and result from synchronous oscillation of liquid at the tray perforations. The oscillation frequency increases as hole velocity increases, as hole spacing decreases and at greater liquid heights on the tray, and can be predicted by correlation. The controlling mechanism is that of a Helmholtz resonator, in which an oscillating mass of liquid near to each perforation cyclically exchanges kinetic energy with potential energy (head) in the bulk liquid across the tray. The rate of exchange is limited by the wave speed in the bulk liquid. The bending stiffness of trays decreases as the free area 
increases, reducing the resonant frequency. We can use effective elastic constants calculated for a perforated plate to identify the vibration frequencies of tray designs which include supports and beams. A typical tray for a column of diameter several metres shows many resonant frequencies in the range $(25-70 \mathrm{~Hz})$ where pressure pulsations might be expected. Stress is amplified close to tray perforations, and fatigue cracks running from tray supports to the nearest hole and from hole to hole are a serious risk during vibration. More experimental work is needed, particularly with valve trays, and with different vapour/liquid systems, to confirm and extend the validity of our approach.

\section{Nomenclature}

$A_{h} \quad$ hole area on tray

$A_{p} \quad$ area of plate

$A_{p h} \quad$ Area of perforated plate associated with a single hole

Bo Bond number, $\rho_{L} g d^{2} / \gamma$

c wave velocity

d hole diameter

$f \quad$ frequency

Fr Froude number, $U\left(g H_{L}\right)^{-0.5}$

$g$ acceleration due to gravity

$H_{L} \quad$ height of liquid

$L \quad$ characteristic dimension of the oscillation

$L_{H} \quad$ composite length scale in Helmholtz oscillation

$N \quad$ number of holes in plate

$P \quad$ mean pressure

$p \quad$ r.m.s. variation in pressure

St Strouhal number, $f d / U$

$s \quad$ hole pitch (distance between centres of adjacent holes)

$s_{m} \quad$ mean hole pitch calculated from equation (7)

$U \quad$ mean velocity of gas through hole

$U_{\text {crit }}$ value of $U$ at which total tray pressure drop is independent of $U$

$u \quad$ variation in hole velocity

$\checkmark \quad$ volume of inter-tray space

We Weber number, $\rho_{G} d U^{2} / \gamma$

\section{Greek symbols}

$\alpha \quad$ fraction of the plate that is uniformly perforated

$\gamma \quad$ surface tension 


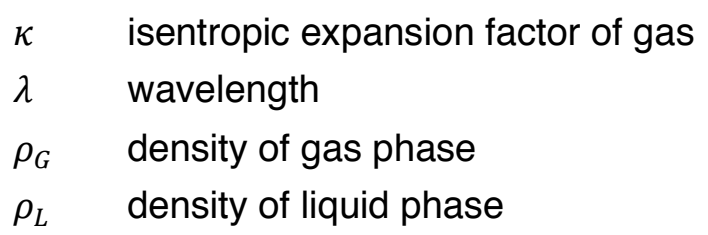

\section{References}

Brierley, R.J.P., Whyman, P.J.M. and Erskine, J.B., 1979, "Flow induced vibration of distillation and absorption column trays" in Distillation 1979, IChemE Symp. Ser No 56, 2.4/45

Brown, R.S., 1958, "Bubbling from perforated plates" PhD thesis University of California, Berkeley

Chan, B.K.C. and Prince, R.G.H., 1966, "Dynamic characteristics of perforated distillation plates operating at low loads" AIChE Journal 12, 232237

Dassault Systèmes S.A., 2014, SOLIDWORKS. Description available at: http://www.solidworks.co.uk/sw/products/simulation/vibration-analysis.htm (Accessed 1 November 2014)

Geipel, C. and Wolgast, A., 2014, Influence of tray geometry and hydraulic conditions on vibration induced damages of sieve and valve trays, Proceedings of Distillation \& Absorption 2014, Friedrichshafen, 14-17 September 2014, p. 113-118.

Kupferberg, A. and Jameson, G.J., 1970, "Pressure fluctuations in a bubbling system with special reference to sieve plates" Trans IChemE 48, T140-T150 Lamb, H., 1932, Hydrodynamics, $6^{\text {th }}$ edition, Cambridge University Press, Cambridge UK Lockett, M.J., 1986, Distillation tray fundamentals, Cambridge University Press, Cambridge UK Mei, C.C., 1989, The Applied Dynamics of Ocean Surface Waves, $1^{\text {st }}$ edition, World Scientific Publishing Co, Singapore Morse, P.M. and Ingard, K.O., 1968, Theoretical Acoustics, McGraw-Hill Co., New York

Muller, R.L. and Prince, R.G.H., 1972, "Regimes of bubbling and jetting from submerged orifices" Chem. Eng. Sci. 27, 1583-1592 Priestman, G.H. and Brown, D.J., 1988, Flow-induced vibration and damage in sieve tray columns, Chem. Eng. Commun. 63, 181-192 Priestman, G.H., Brown, D.J. and Kohler, H.K., 1979, Pressure pulsations in sieve-tray columns in Distillation 1979, IChemE Symp. Ser No 56, 2.4/45 Priestman, G.H., Brown, D.J., 1981, The mechanism of pressure pulsations in sieve-tray columns, Trans IChemE 59, 279-282 
Priestman, G.H., Brown, D.J., 1988, Flow-induced vibration and damage in sieve-tray columns, Chem Eng Communications 63, 181-192

Summers, D.R., Bad Vibrations, Hydrocarbon Engineering, March 2005, 5154

Winter, J.R., 1993, Avoid vibration damage to distillation trays Chem. Eng. Progress 89(5), 42-47

Timoshenko S.P. and Goodier J.N., 1970, Theory of Elasticity, $3^{\text {rd }}$ edition, McGraw-Hill

Waddington, W., Vibration excitation of sieve tray columns by bubbling, MEng Thesis, Sheffield University

Waddington, W, Kohler, H.K. and Brown, D.J., (1974) Vibration excitation of sieve plate columns by bubbling, Trans IChemE 52, 381-383

Zhang, W., Taylor, P.H. and Darton, R.C., 2014, The destructive vibration of column trays, Proceedings of Distillation \& Absorption 2014, Friedrichshafen, 14-17 September 2014, p. 588-593. 


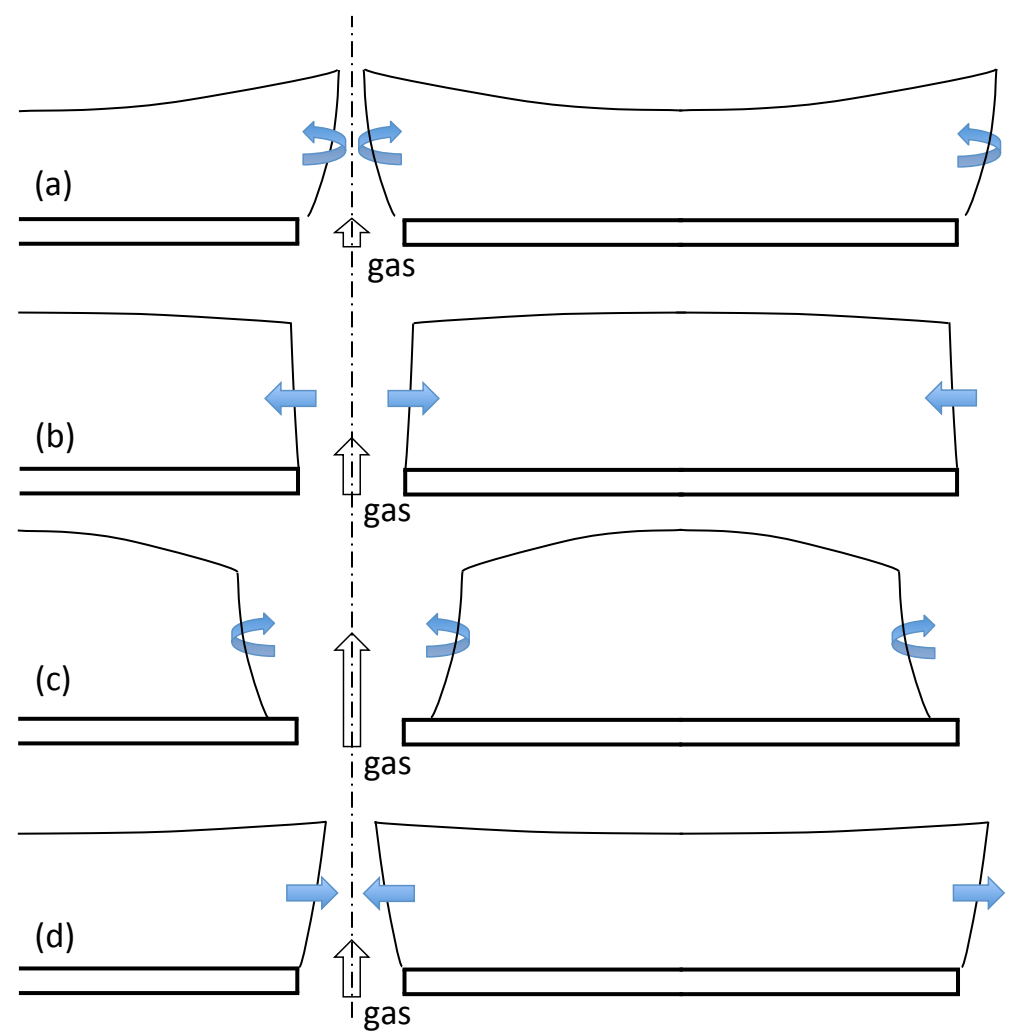

Figure 1 Oscillation of liquid at a tray perforation, and between perforations (not drawn to scale) 


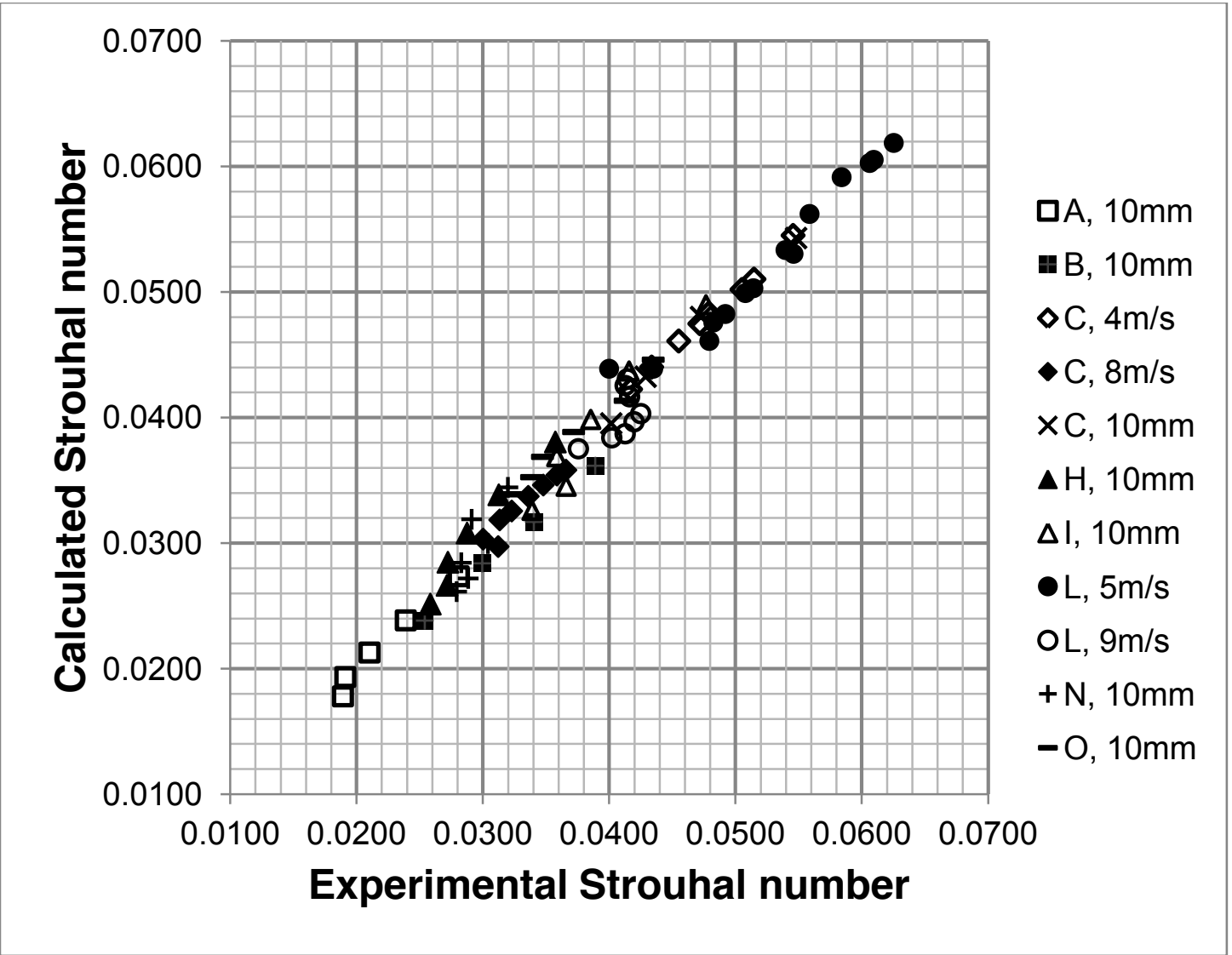

Figure 2. Correlation of pulsation frequency. Strouhal number calculated from equation (6) Data of Priestman et al. (1979) 


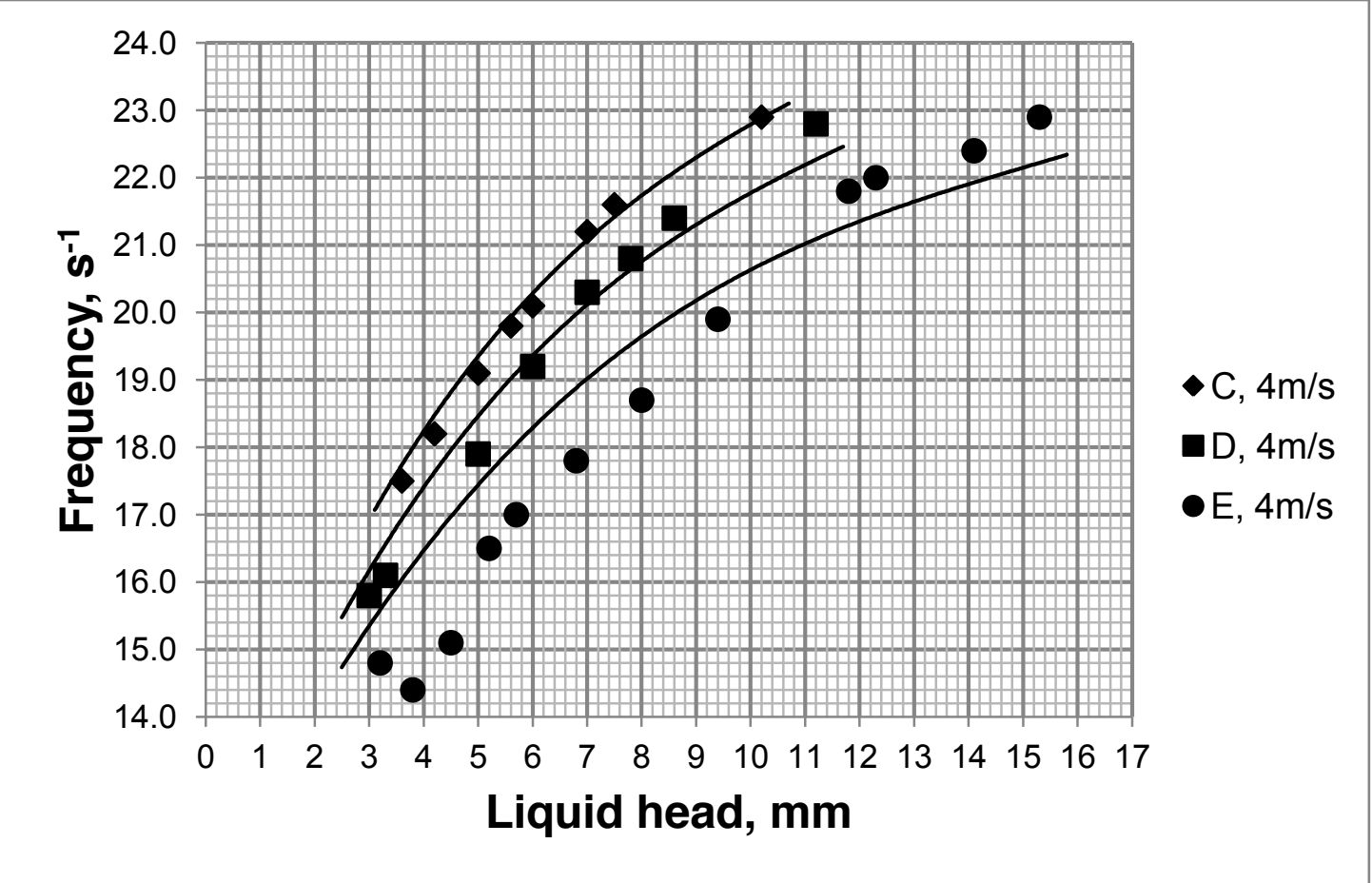

Figure 3. Pulsation frequency for Trays $\mathrm{C}, \mathrm{D}$ and $\mathrm{E}$, at $\mathrm{U}=4 \mathrm{~m} / \mathrm{s}$ : data of Priestman et al. (1979), and calculated from equation (6) 


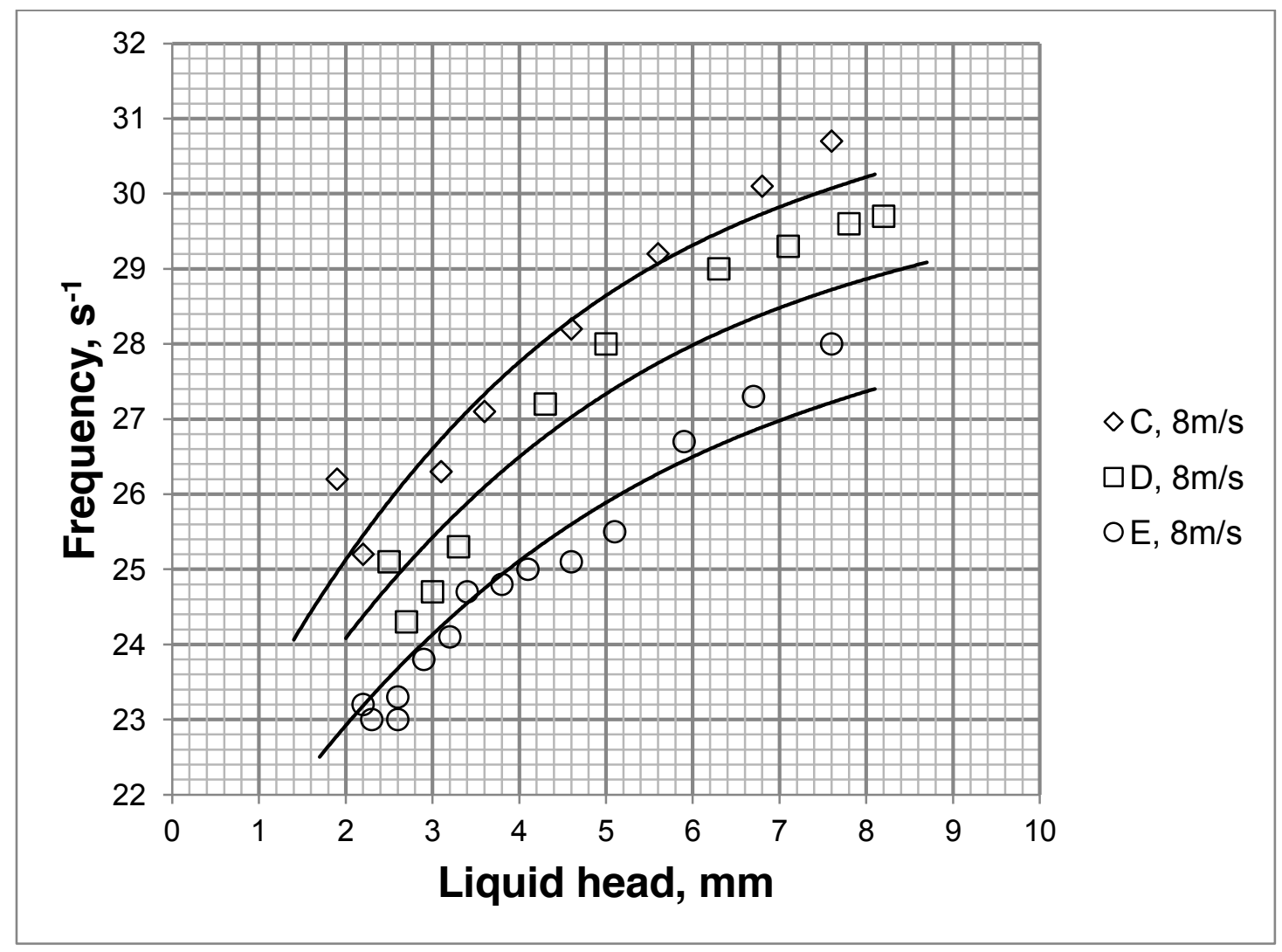

Figure 4. Pulsation frequency for Trays $C, D$ and $E$, at $U=8 \mathrm{~m} / \mathrm{s}$ : data of Priestman et al. (1979), and calculated from equation (6) 


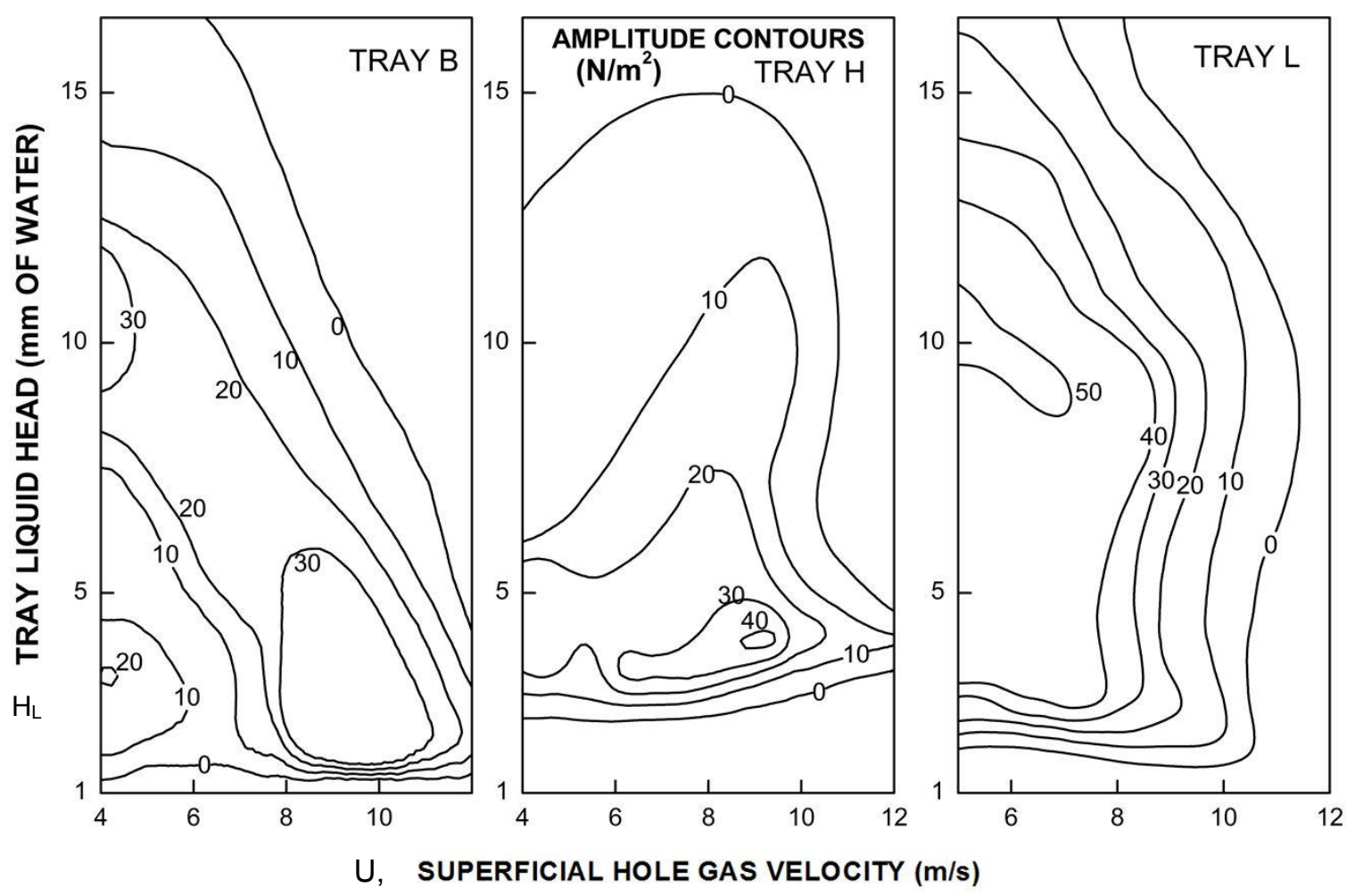

Figure 5. Pressure pulsation fundamental r.m.s. amplitude plots (data of Priestman et al., 1979) 


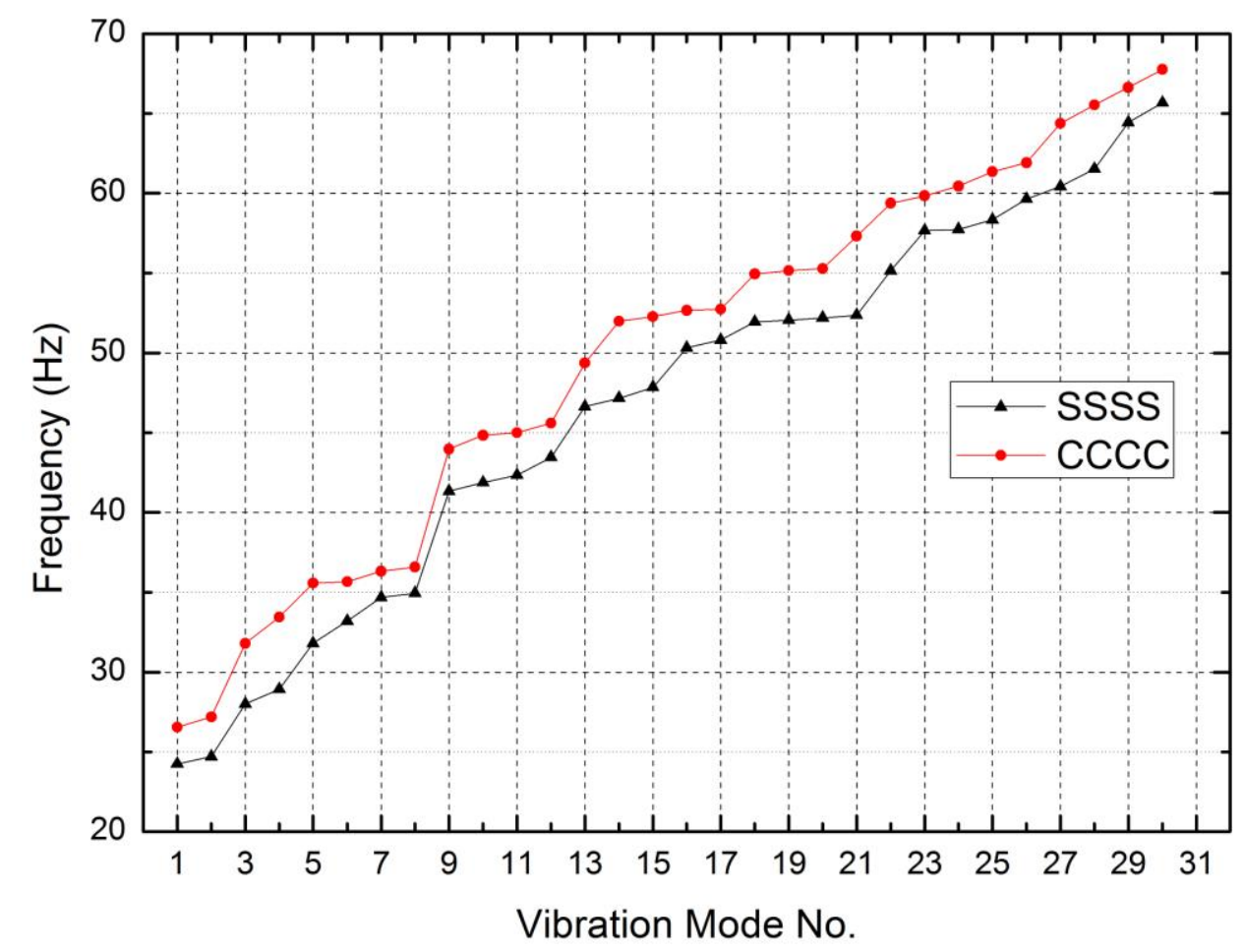

Figure 6. Structural vibration resonance frequencies of idealized tray structure, first thirty modes (Zhang et al., 2014) 


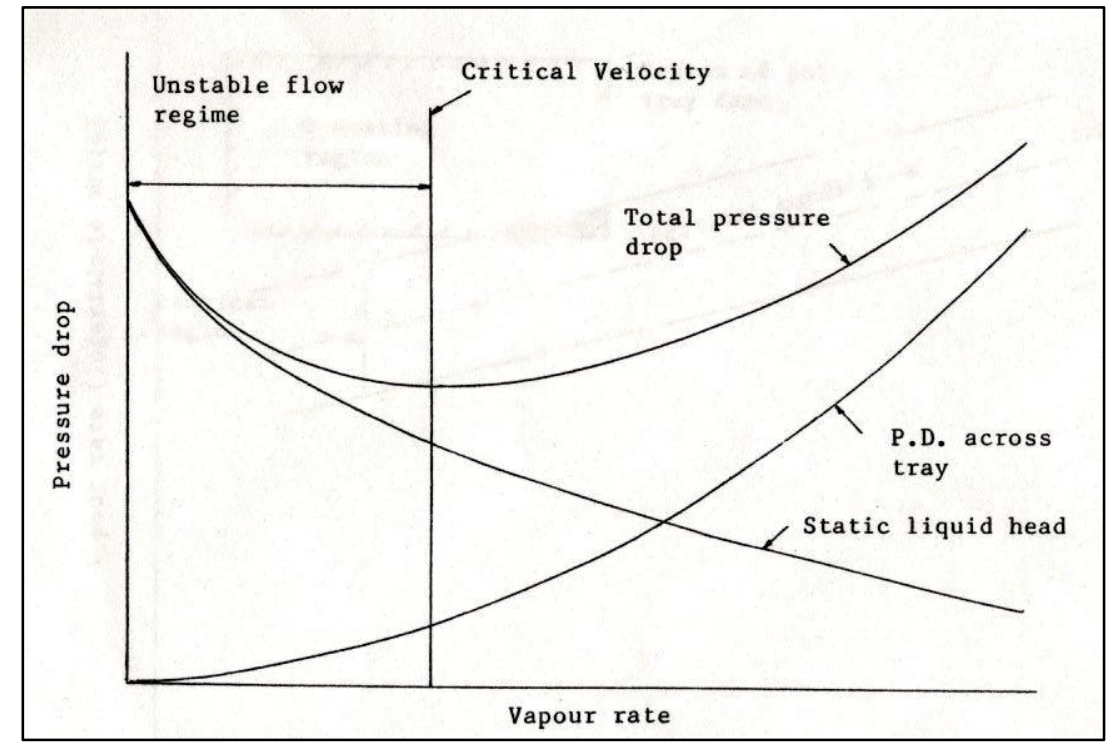

Figure 7. Idealised pressure drop characteristic, from Brierley et al. (1979, Fig. 2) 


$\begin{array}{lcccccccc}\text { Tray } & \mathrm{A} & \mathrm{B} & \mathrm{C} & \mathrm{H} & \mathrm{I} & \mathrm{L} & \mathrm{N} & \mathrm{O} \\ \text { Number of holes } & 403 & 391 & 403 & 108 & 104 & 108 & 38 & 38 \\ \text { Hole diameter, mm } & 4.76 & 6.35 & 9.53 & 9.53 & 12.3 & 15.87 & 12.3 & 15.87 \\ \text { Hole pitch, mm } & 19.05 & 19.05 & 19.05 & 38.1 & 38.1 & 38.1 & 66.0 & 66.0 \\ \alpha & 0.763 & 0.740 & 0.763 & 0.818 & 0.787 & 0.818 & 0.863 & 0.863 \\ s_{m}, \mathrm{~mm} & 20.29 & 20.42 & 20.29 & 39.97 & 40.31 & 39.97 & 68.38 & 68.38\end{array}$

Table 1. Tray details for eight perforation geometries. Rectangular tray 410x405 mm, Priestman et al. (1979). 


$\begin{array}{lccc}\text { Tray } & \text { C } & \text { D } & \text { E } \\ \text { Number of holes } & 403 & 256 & 108 \\ \text { Hole diameter, mm } & 9.53 & 9.53 & 9.53 \\ \text { Hole pitch, mm } & 19.05 & 19.05 & 19.05 \\ \alpha & 0.763 & 0.485 & 0.204 \\ s_{m}, \mathrm{~mm} & 20.29 & 22.11 & 24.55\end{array}$

Table 2. Tray details for tray series $C, D$ and $E$ with reducing number of holes. Rectangular tray 410x405 mm, Priestman et al. (1979). 